\title{
Risk assessment in ambulatory surgery: challenges and new trends
}

\author{
L. Reuven Pasternak MD MBA MPH
}

$\mathrm{T}$ HE explosive growth of ambulatory surgical programs has been one of the most formidable changes in the practice of medicine in the past quarter of century. Few other events have affected so many patients in such a fundamental fashion. It has been estimated that approximately 60 to $70 \%$ of all surgical procedures performed in the United States are now being done on an outpatient basis. ${ }^{1}$ As outpatient surgery continues to diffuse to include a wide range of procedures and be offered to more high-risk patients, it is important to examine its safety in actual settings for high-risk populations.

Although complications in ambulatory surgery are relatively uncommon, little is known about the characteristics of patients or settings that have higher rates of poor outcomes such as death, unplanned hospitalizations or emergency room visits following the procedure. This situation reflects the not uncommon phenomenon of rapid diffusion of a technology without an evidence based analysis to determine appropriate risk factors for analysis. While much has been made of the preoperative process as a vehicle for appropriate testing, the ability to truly determine the relative risk of ambulatory surgery is, at best, ambiguous despite over 20 years into its rapid growth phase.

\section{ASA classification}

The first attempt to quantify risks associated with surgery and anesthesia was undertaken by Meyer Saklad ${ }^{2}$ in 1941 at the request of the American Society of Anesthesiologists. This effort was the first by any medical specialty to stratify risk for its patients. Saklad's system was based on mortality secondary to anesthesia due to associated preoperative medical conditions. Type of anesthesia and nature of surgery were not considerations in this system and the divisions were based on empirical experience rather than on specific sets of data and reflect the techniques and standards of practice as of $\mathbf{5 0}$ years ago. Four preanesthesia risk categories were established ranging from category 1 (least likely to die) to category 4 (highest expectation of mortality).

The current ASA system (Figure 1 ) is a modification of this work, adding an additional fifth category for moribund patients undergoing surgery in a desperate attempt to preserve life. Numerous studies have demonstrated an association of mortality with ASA class independent of anesthetic technique. ${ }^{2-12}$ However, this information has limited application as it relates to mortality as its sole outcome and is based on anesthetic techniques as practiced more than 20 years ago. Apfelbaum ${ }^{3}$ and Meridy, ${ }^{4}$ for example, have noted a lack of correlation between ASA status and cancellations, unplanned admissions and other perioperative complications in outpatient surgery. It should also be noted that the original study of Saklad and subsequent derivations were not based on actual determinations of mortality and associated morbidity and mortality, either retrospectively or prospectively. Thus, while useful as a broad assessment of preoperative medical status, the current ASA classification is limited in its ability to truly establish risk or serve as a basis for formulating clinical guidelines without an associated risk index for the surgical procedure.

\section{The Johns Hopkins risk classification system}

The Johns Hopkins risk classification system ${ }^{5}$ (Figure 2 ) was one of the first attempts to formulate a multifactorial risk assessment system by adding the invasiveness of the surgical procedure as a function of risk along with the more traditional preoperative medical condition. The Johns Hopkins risk classification system is based on the well established assumption that the nature of the surgery is clearly a major determinant of risk and needs to be coordinated with medical status in determining preoperative risk assessment. This system is predicated on the assumption that patients of identical medical status undergoing minor office procedures are at less risk of adverse events and in need of less preparation than those undergoing

From the Department of Anesthesiology, The Johns Hopkins University School of Medicine, Baltimore, Maryland, USA.

Address correspondence to: Dr. L. Reuven Pasternak, The Johns Hopkins Bayview Medical Center, Pavilion 01-1-22, 4940 Eastern Avenue, Baltimore, Maryland 21224-2780, USA. Phone: 410-550-6968; Fax: 410-550-6966; E-mail: rpastern@jhmi.edu 
surgery that entail blood loss, fluid shifts, or other significant physiologic intervention and compromise. Such an assumption matches a common sense approach assumed by clinicians in virtually all types of settings and daily practice. While various systems exist for stratifying surgical and medical risk in an intensive care setting, ${ }^{3,4,7-9}$ these have several drawbacks when applied in a general preoperative setting, especially for outpatient procedures.

This system was built upon the observations of the confidential inquiry into perioperative deaths in Great Britain as reported in $1987 . .^{10}$ In that retrospective study it was determined that surgical condition was most frequently cited as the cause of death, followed by medical status and then anesthesia (Figure 3 ). It also proposed that mortality was most often a combination of factors rather than assignment to a single specific cause. The nature of surgical causation was not established with regard to invasiveness, site of surgery or other factors. The Johns Hopkins risk classification system proposed that the risk of surgery is a combination of several factors, including invasiveness, associated blood loss and fluid shift, entry into specific body areas (e.g., intrathoracic, intracranial), postoperative anatomic and physiologic alterations and need for postoperative intensive care monitoring. Procedures were assigned to the various categories in consultation with the surgical, anesthesia, medical and nursing staffs by which these patients are managed. Though broad in their scope and subject to potential variances in interpretation, the five categories may provide a reasonable basis for use in the practice environment, pending further verification. However, like the ASA, this system is based on assumptions and presumptions rather than actual fact.

\section{ASA advisory on preoperative evaluation}

When the ASA Preoperative Advisory Task Force began its deliberations in 1994, it agreed, during its initial phase, that the ability to determine appropriate risk, for purposes of patient testing and consultation, needed to include medical condition and the nature of the surgical procedure. The Task Force participants determined that an algorithm might be used that may fit most situations relating to the timing of the preanesthesia evaluation (Figure 4). The Task Force adopted a system that included two levels of stratification for the medical status and three for the nature of the surgical procedure. In doing so, it was using an algorithm based on the same type of flow as that adopted by the American Heart Association/American College of Cardiology guidelines and is part of the model adopted by the ASA Preanesthesia Task Force. On the basis of this algo- rithm, it was determined that healthy patients undergoing procedures of minor or intermediate complexity or stable patients with significant medical issues but with procedures of low risk or complexity may have their evaluation on the day of surgery with the caveat that appropriate information is available for review prior to surgery to provide reasonable assurance of a patient who is sufficiently prepared for surgery or anesthesia. This recommendation did not preclude the possible benefit of a preanesthesia evaluation prior to the day of surgery for such purposes as education, allaying anxiety, or prevention of day of surgery delays associated with last minute evaluation It simply referred to the safety of this timing of the evaluation should all parties be comfortable with it.

Conversely, patients with high medical risk having complex procedures were believed to benefit from a preanesthesia evaluation prior to the day of surgery. Exceptions to this mandate were to be made on the basis of the anesthesiologist's comfort with the nature of information provided prior to the day of surgery and the ability to appropriately prepare the patient without such a visit. However, again, this system represented a consensus of opinion rather than an actual evidence based model of appropriate risk factors and outcomes. In fact, the ASA in its report noted the paucity of appropriate data on which to base such a system and strongly advocated for more definitive studies.

\section{Challenges from the past - and more}

The ability to determine risk by measures of admission and death for ambulatory surgery are difficult due to the low frequency of events. Such a study would most certainly have to be multi-institutional and, perhaps, national in scope to capture the relevant variables to allow for a true determination of the appropriate risks associated with this endeavour. To add to the complexity of such an analysis, additional factors have come into play as confounding variables in this enterprise. Beyond the issue of medical status and type of surgery the concern of site of surgery has now come forward. As with the issue of the proliferation of procedures done in the absence of controlled studies to assess outcome, ambulatory surgery locations have expanded beyond the traditional hospital to freestanding multispecialty centres, single specialty centres and physician offices. The often cited episodic cases of deaths in physician office locations for cosmetic procedures has brought forward much attention but little objective insight into the issue of the extent to which the factors of type of surgery, medical status, anesthesia, and location of procedure interact to create a true risk assessment structure for the tens of millions of 
TABLE I Number of procedures and \% of total performed by location of care in 5\% Medicare sample for 1994-1999

\begin{tabular}{lllll}
\hline Procedure & Outpatient hospital & ASC & Office & \% Outpatient* \\
\hline Cataract extraction & 256087 & 163032 & 15917 & 98 \\
TURP & 4822 & 353 & 322 & 16.1 \\
Inguinal hernia repair & 25690 & 2727 & 312 & 74.5 \\
Laparoscopic cholecystectomy & 14336 & 200 & 171 & 39.5 \\
D\&C & 6100 & 779 & 1127 & 80.5 \\
Simple mastectomy & 718 & 51 & 14 & 32 \\
Modified radical mastectomy & 1636 & 43 & 45 & 17 \\
Carpal tunnel repair & 13862 & 2860 & 442 & 92.1 \\
Knee arthroscopy & 18203 & 3222 & 353 & 88.5 \\
Femoral hernia repair & 821 & 76 & 19 & 24.9 \\
Hysteroscopy & 5178 & 799 & 651 & 86.2 \\
Shoulder cuff repair & 2982 & 319 & 50 & 48.3 \\
Umbilical hernia repair & 3271 & 273 & 59 & 54.1 \\
Arteriovenous graft placement & 3253 & 136 & 35 & 32.5 \\
Hemorrhoidectomy & 3391 & 360 & 4415 & 89.9 \\
Hysterectomy, vaginal & 114 & 6 & 48 & 2.7 \\
All procedures & 360464 & 175236 & 27980 & \\
\hline
\end{tabular}

* Represents the total procedures performed in the outpatient hospital + ambulatory surgery centre (ASC) + office of the total number of such procedures performed in all locations of care during the same time period. TURP $=$ transurethral resection of the prostate; $\mathrm{D} \& \mathrm{C}=$ dilatation and curetage.

ambulatory procedures performed annually in North America alone. During the late 1990s, office-based surgical procedures became much more common, with an estimated 5 to $8 \%$ of procedures being performed in the office in the year $2000 .{ }^{11}$

A recently published study by Fleisher $e t a l .12$ was one of the first to address this issue. The authors utilized a 5\% sample of Medicare beneficiaries from 1994-1999, matching part A and part B data to obtain both facilities and medical provider data. Patients undergoing any one of 16 surgical procedures were identified with outcome of death, hospital admission, or emergency room visit measured. The selection of surgical procedures was based upon their prevalence in the outpatient setting and their rapid diffusion from the inpatient to outpatient setting in the last ten years. Death, hospitalizations and emergency room (ER) visits on the day of surgery (calendar day), within seven days, and within 30 days of the procedure were the three outcome variables. There were 563,680 surgical procedures identified that were further stratified by site of surgery: hospital-based outpatient $(360,464)$, free standing ambulatory centre (ASC) $(175,236)$, and office-based $(27,980)$ (Table I).

For the 16 procedures, there was a trend towards increasing frequency in the outpatient setting from 1994 to 1999, except for cataract surgery which was already performed in the outpatient setting $98.6 \%$ of the time in 1994 (Table I). For all 16 procedures, the proportion of surgeries in the outpatient hospital increased from $80.6 \%$ in 1994 to $88.2 \%$ in 1999 . This is higher than the rate for all surgical procedures because our select criterion allowed only procedures that could be performed in the outpatient setting.

Table II shows the rates of death, ER visits, and inpatient admissions at seven and 30 days post-surgery. There were no deaths on the day of surgery in the office, four deaths in the ASC $(2.3$ per 100,000$)$ and nine in the outpatient hospital $(2.5$ per 100,$000 ; P=$ NS) Within seven days of surgery, there were ten deaths in the office (36 per 100,000), 43 deaths in the ASC (25 per 100,000) and 179 deaths in the outpatient hospital (50 per 100,000; $P<0.05$ for ASC compared to outpatient hospital) The number of ER visits within seven days of surgery was $248(879$ per 100,000$)$ in the office, 1,453 (829 per 100,000$)$ in the ASC and 7,477 $(2,074$ per 100,000$)$ in the outpatient hospital $(P<$ 0.05 for outpatient hospital compared to either ASC or office) The number of patients admitted to an inpatient hospital within seven days was $511(1,826$ per 100,000$)$ in the office, $1280(730$ per 100,000) in the ASC and $12,489(3,464$ per 100,000$)$ in the outpatient hospital $(P<0.05$ for all groups compared to each other). The rates per day at zero to seven days and eight to 30 days for the three outcome variables were also calculated (Table II). The rate of deaths per day was lower during the first seven days after surgery when compared to the subsequent 23 days, while the rate of ER visits and inpatient admissions per day were greatest during the first seven days (Table II). 
TABLE II Rate of adverse events per day (per 100,000 procedures) by site of care for 16 procedures performed in Medicare beneficiaries from 1994-1999

\begin{tabular}{lllll}
\hline Adverse events & Outpatient hospital & ASC & Office & Overall outpatient \\
\hline Death same calendar day as procedure & 2.5 & 2.3 & & 2.3 \\
Death 0-7 days & 6.2 & 3.1 & 4.5 & 5.1 \\
Death 8-30 days & 7.3 & 5.6 & 5.2 & 6.6 \\
Death 0-30 days & 7.0 & 4.9 & 5.1 & 6.3 \\
ER visit, 0-7 days & 259.3 & 103.6 & 109.9 & 203.3 \\
ER visit, 8-30 days & 106.6 & 79.6 & 60.3 & 95.9 \\
ER visit 0-30 days & 139.0 & 82.9 & 69.9 & 118.1 \\
Inpatient admission, 0-7 days & 433.1 & 91.3 & 228.3 & 316.3 \\
Inpatient admission, 8-30 days & 115.3 & 74.0 & 74.3 & 100.4 \\
Inpatient admission 0-30 days & 174.5 & 70.2 & 101.0 & 138.4 \\
Total procedures & 360464 & 175236 & 27980 & 563680 \\
\hline
\end{tabular}

$\mathrm{ER}=$ emergency room, ASC $=$ ambulatory surgery centre.

TABLE III Event rates and total cases for basic demographic and location of care factors for Medicare beneficiaries undergoing 15 procedures during 1995-1999 used for logistic regression analysis

\begin{tabular}{|c|c|c|c|c|c|c|c|}
\hline & $\begin{array}{l}\text { Inpatient } \\
\text { admission }\end{array}$ & ER visit & Death & Total cases & $\begin{array}{l}\text { Inpatient admission } \\
\text { rate per } \\
100,000 \\
\text { procedures } \\
0-7 \text { days }\end{array}$ & $\begin{array}{l}\text { ER visit } \\
\text { rate per } \\
100,000 \\
\text { procedures } \\
0-7 \text { days }\end{array}$ & $\begin{array}{l}\text { Death } \\
\text { rate per } \\
100,000 \\
\text { procedures } \\
0-7 \text { days }\end{array}$ \\
\hline Male & 5,529 & 3,746 & 102 & 181,723 & 3,043 & 2,061 & 56 \\
\hline $\begin{array}{l}\text { Female } \\
\text { Age }\end{array}$ & 6,603 & 4,759 & 104 & 301,857 & 2,187 & 1,577 & 34 \\
\hline $65-69$ & 2,212 & 1,559 & 31 & 80,782 & 2,738 & 1,930 & 38 \\
\hline $70-74$ & 2,951 & 2,083 & 45 & 121,642 & 2,426 & 1,712 & 37 \\
\hline $75-79$ & 3,027 & 2,127 & 54 & 129,077 & 2,345 & 1,648 & 42 \\
\hline $80-84$ & 2,292 & 1,610 & 39 & 93,921 & 2,440 & 1,714 & 42 \\
\hline $85+$ & 1,650 & 1,126 & 37 & 58,158 & 2,837 & 1,936 & 64 \\
\hline Office & 401 & 209 & 8 & 23,225 & 1,727 & 900 & 34 \\
\hline Outpatient hospital & 10,281 & 6,384 & 156 & 303,696 & 3,385 & 2,102 & 51 \\
\hline ASC & 1,058 & 1,256 & 36 & 150,519 & 703 & 834 & 24 \\
\hline
\end{tabular}

$\mathrm{ER}=$ emergency room, $\mathrm{ASC}=$ ambulatory surgery centre.

The absence of any large scale evaluation of the safety of outpatient surgery has led some authors to estimate that the rate of operative mortality associated with anesthesia and surgery in the outpatient setting (either in the operating room or postanesthesia care unit) is of the order of 0.25 to 0.5 per 100,000 outpatient surgeries. ${ }^{9}$ These estimates were based upon insurance claims of intraoperative mortality related to anesthesia in healthy individuals undergoing elective inpatient surgery ${ }^{10}$ and have questionable applicability to the fastest growing surgical population in the United States - the rapidly expanding geriatric population. The study of Fleisher et al. calculated the sameday mortality rate in a population over age 65 to be 2.5 per 100,000 or five to ten times greater than these estimates. Further, as seen in Table III, age alone does not appear to be an independent risk factor once the patient population enters into the geriatric grouping until age 85 , versus the traditional and unsubstantiated age 70 used for the current classification systems.

The influence of location of care varied by procedure. For those models with sufficient sample size, the risk-adjusted odds ratio for hospitalization and death within seven days for office-based care is shown in Table IV. Hemorrhoidectomy in the office was associated with a significantly lower risk-adjusted rate of adverse events compared to ASCs, while cataract surgery, hysteroscopy, inguinal hernia repair, arterio-venous $(\mathrm{A}-\mathrm{V})$ graft placement, knee arthroscopy, transurethral resection of the prostate (TURP) and umbilical hernia repair were associated with significantly higher rates, frequently higher than outpatient hospitals. 
TABLE IV Increased risk associated with office care compared to the ASC for a given procedure when each risk procedure was evaluated individually

\begin{tabular}{llll}
\hline Effect & $\begin{array}{l}\text { Odds ratio for } \\
\text { increased risk of } \\
\text { office care compared } \\
\text { to ASC }\end{array}$ & $\begin{array}{l}\text { 95\% Wald } \\
\text { confidence limits }\end{array}$ \\
\hline Hemorrhoidectomy & 0.146 & 0.076 & 0.281 \\
Cataract extraction & 1.555 & 1.288 & 1.877 \\
Hysteroscopy & 2.313 & 1.094 & 4.890 \\
Inguinal hernia repair & 3.818 & 2.335 & 6.243 \\
A-V graft placement & 4.046 & 1.580 & 10.361 \\
Knee arthroscopy & 4.718 & 2.472 & 9.005 \\
TURP & 7.491 & 4.162 & 13.481 \\
Umbilical hernia repair & 10.793 & 3.731 & 31.223 \\
\hline
\end{tabular}

*Adjusted for age, gender, race and prior admission history. A-V $=$ arterio-venous; TURP $=$ transurethral resection of the prostate

\section{Conclusion}

There is a great deal of interest in safety in health care, and the surgical suite is perhaps one of the greatest areas of interest due to the potential for life threatening risk. Nonetheless, the availability of appropriate risk assessment strategies is but in its infancy despite an exponential growth of ambulatory surgery. While there is reason to believe this growth has been conducted in a safe fashion, the increasing acuity of patients and procedures performed in a multiplicity of settings reinforce the need for a scientific determination of risk to aid clinicians and policy makers alike in their work.

\section{References}

1 Owings MF, Kozak LJ. Ambulatory and inpatient procedures in the United States, 1996. National Center for Health Statistics. Vital Health Stat 1998; 13.

2 Saklad $M$. Grading of patients for surgical procedures. Anesthesiology 1941; 2: 281-4.

3 Apfelbaum JL. Preoperative evaluation, laboratory screening, and selection of adult surgical outpatients in the 1990s. Anesthesiol Rev 1990; 17: 4-12.

4 Meridy $H W$. Criteria for selection of ambulatory surgical patients and guidelines for anesthetic management: a retrospective study of 1553 cases. Anesth Analg 1982; 61: 921-6.

5 Pasternak LR. Preoperative evaluation of the ambulatory surgery patient. Ambulatory surgery.

Anesthesiology Report 1990; 3: 8.

6 Dripps RD, Lamont A, Eckenhoff JE. The role of anesthesia in surgical mortality. JAMA 1961; 178: 261-6.

7 Marx GF, Mateo CV, Orkin LR. Computer analysis of postanesthetic deaths. Anesthesiology 1973; 39: 54-8.
8 Carter DC, Campbell D. Evaluation of the risks of surgery. Br Med Bull 1988; 44: 322-40.

9 Arens JF. Assessment and reduction of cardiovascular anesthetic risk. Anesth Analg 1989; 52-6.

10 Fowkes SC, Fowkes FGR, Lunn JN, Robertson IB, Samuel P. Epidemiology in anaesthesia III: factors affecting mortality in hospitals. Br J Anaesth 1982; 54: 811-6.

11 Grazer FM, de Jong RH. Fatal outcomes from liposuction: census survey of cosmetic surgeons. Plast Reconstr Surg 2000; 105: 436-46.

12 Fleisher LA, Pasternak LR, Herbert R, Anderson GS. Admission and death after outpatient surgery in the elderly: the importance of the patient, system, and location of care. Arch Surg 2004; 139: 67-72. 